\title{
El coaching y el desempeño laboral en el sector cooperativo de la provincia de Tungurahua
}

\section{Coaching and work performance in the cooperative sector of the province of Tungurahua}

Elias David Caisa Yucailla

María Dolores Guamán

Rodrigo Fernando Miranda López

Universidad Técnica de Ambato, Ecuador

Autor para correspondencia: eliasdcaisa@uta.edu.ec,md.guaman@uta.edu.ec, rf.miranda@uta.edu.ec

Fecha de recepción: 08 de Julio 2017 - Fecha de aceptación: 15 de Enero de 2018

Resumen: "La Cooperativa de Ahorro y Crédito Cámara de Comercio de Ambato Ltda.", (CCCA) es una institución financiera que brinda productos y servicios financieros con la más alta calidad, promoviendo el desarrollo socioeconómico de la comunidad, cuenta con recursos humanos capaces y motivados para construir una organización sólida, rentable y segura. Siendo una entidad que profundiza el proceso de constitución de un sistema económico, social y solidario, en el que los seres humanos son el fin, una institución que debe tomar acciones estratégicas para mejorar de una manera exitosa su crecimiento. Es por esta razón que el presente trabajo de investigación se ha enfocado en realizar un análisis a los socios y empleados de la cooperativa, con el fin de establecer las estrategias necesarias que permitan mejorar el desempeño laboral en sus empleados con la implementación del coaching. Los datos arrojados por esta investigación de campo aplicada a los empleados y socios de la cooperativa indican que es importante implementar un programa de coaching que según los estudios realizados ayudara a mejorar el desempeño laboral de los empleados de la institución. Es así que un programa de coaching en la CCCA se direcciona a mejorar el desempeño laboral, de los empleados y de todas aquellas personas que forman parte de la institución y que miran un futuro de crecimiento para las instituciones financieras.

Palabras Claves: cooperativa; coaching; desempeño; eficiencia

Abstract: "The Savings and Credit Cooperative Chamber of Commerce Ambato Ltda." (CCCA) is a financial institution that provides financial products and services with the highest quality, promoting the socio-economic development of the community, has capable and motivated human resources. To build a solid, profitable and secure organization. Being an entity that deepens the process of constitution of an economic, social and solidary system, in which human beings are the end, an institution that must take strategic actions to improve its growth in a successful way. It is for this reason that the present research work has focused on an analysis to the members and employees of the cooperative, in order to establish the necessary strategies to improve the work performance in their employees with the implementation of coaching The data provided by this field research applied to employees and members of the cooperative indicate that it is important to implement a coaching program that, according to the studies, will help improve the work performance of the institution's employees. Therefore, a coaching program in the CCCA is aimed at improving the work performance of employees and all those people who are part of the institution and who are looking at a future of growth for financial institutions.

Key Words: cooperative; coaching; performance; efficiency 


\section{Introducción}

En las cooperativas de Ahorro y Crédito de la provincia de Tungurahua existe un déficit cumplimiento de metas en lo referente a la administración de las mismas, se ha visto que existen muchas instituciones financieras que han sido intervenidas y en otras ocasiones cerradas, ya sea por falta de planificación estratégica, mala tomas de decisiones, falta de productividad, entre otros factores externos que afectan al rendimiento de las mismas, es por eso que se quiere desarrollar un procedimiento de coaching que ayude a mejorar el desempeño laboral en sector cooperativista y la comunidad se sienta conforme con el servicio adquirido dentro de cada institución.

El presente trabajo de investigación se estructurará como parte inicial en los conceptos utilizados como son los temas de coaching, la gestión empresarial, el desempeño laboral y cooperativa, y como se relación cada uno de esto. En la metodología utiliza tipos y métodos de investigación empleados para la investigación, se añade la muestra enfocada en los trabajadores y socios de la cooperativa sumando un total de 10213 personas. Dentro de los de resultados se realiza un análisis basado en la relación de las variables empleadas, realizando una regresión lineal, la cual brinda un resultado que indica que aplicar un programa de coaching dentro de la cooperativa seria favorecedor, ya que ayudaría a ampliar los conocimientos dentro de la misma. En la discusión se presenta la aportación del coaching dentro de la organización porque será una herramienta que permita que todos los colaboradores definan bien sus objetivos personales y los trabajen en conjunto con los objetivos generales de la cooperativa buscando así un cambio importante en los colaboradores que permita obtener soluciones de manera colectiva. Como último punto las conclusiones a las cuales se ha llegado, básicamente demuestran que la aplicación de un programa de coaching dentro de la empresa, beneficiaria en gran magnitud porque permite el cambio de perspectivas de los trabajadores y aumenta el compromiso de responsabilidad que se traduce en una mejora colectiva dentro de la cooperativa.

\section{El Coaching}

Coaching, por definición, implica crear una relación muy cercana y personal con el cliente, creyendo en las personas y en la naturaleza humana, y facilitando en otros la confianza, el cariño, la posición, la composición, la energía, el desarrollo, la disciplina, la exploración, los restos, la retroalimentación y mucho más (Hall y Duval, 2010, p. 19).

El coaching es la habilidad de trabajar con varias personas que permiten obtener resultados fuera de lo normal y por ende aumentar su cometido. Este se fundamenta en una relación donde el coach concurre en la instrucción de novedosas formas de ser y hacer, necesarias para crear un cambio paradigmático o cultural (Talles, 2011).

Se considera de la misma manera al coaching como una metodología eficaz mediante la cual es posible brindar ayuda, a otra persona para que se a realice y desempeñarse hasta el grado más elevado de sus capacidades (Soyentrepreneur, 2013). 
Produce cambios efectivos y duraderos en el emprendedor, en su equipo de labor, en sus negocios, y, en consecuencia, en los resultados económicos de la empresa y/o negocio (Guia del Emprendor , 2016).

El coaching organizacional tiene como finalidad generar cambios en la forma de actuar, de relacionarse y comunicarse de los individuos. Supone a la organización como una red de conversaciones y su efectividad en la comunicación como determinante de su éxito o fracaso (Gestionando la confianza desde el coaching organizacional como medio de prevencion de conflictos, 2012).

Según Suarez (2015) indica que" El coaching tiene el poder de cambiar vidas, de convertirte en una persona exitosa, y todo con herramientas que tú ya tienes dentro de ti” (p.9).

El coaching es la habilidad de observar, escuchar, y hacer preguntas por medio de una buena técnica para lograr que otros descubran sus propios recursos, emprendan acciones y sean capaces de continuar alcanzando objetivos por sí mismo (...) es un proceso de ayuda, escucha activa y toma de conciencia que te llevara a descubrir y maximizar todo tu potencial creativo teniendo en cuenta tus habilidades, circunstancias y recursos para que puedas plantearte mejor metas y alcanzar tus objetivos (Suarez,2015,p. 10).

El proceso de coaching se centraliza en el desempeño, facilita las herramientas para alcanzar los tres propósitos destinados para los gerentes y los supervisores. Radica en cuatro fases a saber: Desarrollo de una dependencia de Sinergia, utiliza los cuatro padrones del coaching fijado en el desempeño: Entrenamiento, resolver, problemas, ajustar el desempeño y mantener el desempeño; desarrollo de trabajadores, administrar premios que construyan el compromiso y fomentar el logro de resultados, en si los coaches ejecutan varias tareas: aconsejan, establecen dirección y dan feedback (Talles, 2011).

Según (Entre el coaching ejecutivo y el coaching de negocios, 2015) el coaching de negocios es un proceso que va dirigido a gerentes generales, gerentes estratégicos, vicepresidentes corporativos; presidentes de directorio o empresarios. Estos directivos tienen niveles de experiencia y desarrollo muy elevados, demostrada capacidad y resultados a lo largo de su vida en el mundo empresarial. Tiene como finalidad ayudar al cliente y a su negocio para lograr optimizar los resultados y obtener mayores ganancias. Esta finalidad abarca el liderazgo, gestión empresarial, y técnicas de administración y técnicas de administración, una mayor base de desempeño, mejores estrategias para poder enfrentar las decisiones internas y a la competencia externa, así como un mayor rendimiento global (Acosta, 2011).

Hay quien ve el coaching como el arte de aplicar una metodología de aprendizaje interpersonal desde el asesoramiento o como el acompañamiento de una persona que asume el papel del coach o guía ente otra que asume el papel de coachee o aprendiz (...) En el ámbito empresarial, se habla de la relación jefe-colaborador como un proceso formativo, que es liderado por un superior inmediato y que actúa como entrenador, con la intención de mejorar el comportamiento de cada individuo en su puesto de trabajo y alcanzar los objetivos (Tovar, 2012, p. 11). De hecho, el coaching, es un enfoque actual e liderazgo que forma parte de la cultura empresarial de las más modernas y prosperas empresas. Trata de delegar personal que ayuden a 
otras en el desarrollo de sus capacidades en el campo empresarial y son una herramienta que permite acompañar y preparar a los futuros líderes mediante estrategias integradoras de todos los aspectos de la persona, para dirigirlos hacia una excelencia organizacional (La Figura del Coaching en el Ambito Laboral, 2004).

\section{Gestión Empresarial}

La gestión empresarial consiste en perseguir y desarrollar nuevas estrategias para enfrentar los desafíos y las necesidades reales del mundo empresarial actual y a la vez optimizar recursos para alcanzar objetivos propuestos.

La gestión empresarial ocupa un lugar importante para el cumplimiento de los objetivos es decir es la base para la determinación y la satisfacción de las metas propuestas en los aspectos políticos, sociales y económicos que reclinan en la competencia que posea el administrador. Según Gonzales (2014) afirma "Es el proceso de toma de decisiones realizado por los órganos de dirección, administración y control de una entidad, basado en los principios y métodos de administración, en su capacidad corporativa".

\section{Desempeño Laboral}

El desempeño laboral es la manera en que los trabajadores ejecutan su labor. Ese se evalúa durante las revisiones de su rendimiento, mediante las cuales el empleador toma en cuenta aspectos como la capacidad de liderazgo, la gestión de tiempo, las habilidades organizativas y la productividad con el fin de analizar a cada empleado individualmente (...) la fijación de metas y objetivos es una forma de garantizar que el desempeño en el trabajo sea aceptable. El cumplimiento de los objetivos diarios dirige a los metas generales puedan mejorar la evaluación del desempeño laboral cuando llegue el momento de llevarla cabo (Hose, s.f.).

El término desempeño laboral se refiere a lo que en realidad hace el trabajador y no solo lo que sabe hacer, por lo tanto le son esenciales aspectos tales como: las aptitudes (la eficiencia, calidad y productividad con que desarrolla las actividades laborales asignadas en un período determinado), el comportamiento de la disciplina, (el aprovechamiento de la jornada laboral, el cumplimiento de las normas de seguridad y salud en el trabajo, las específicas de los puestos de trabajo) y las cualidades personales que se requieren en el desempeño de determinadas ocupaciones o cargos y, por ende, la idoneidad demostrada.

\section{Cooperativas de Ahorro y Crédito}

Las cooperativas de ahorro y crédito son aquellas "cooperativas de servicio que tengan por objeto único y exclusivo brindar servicios de intermediación financiera en beneficio de sus socios". Para ello, entre otras actividades, pueden recibir depósitos de sus socios y de terceros; contraer préstamos con instituciones financieras nacionales o extranjeras y otorgar préstamos a sus socios, que se encuentren amparados por garantía hipotecaria. (BANCAFACIL, 2016).

\section{Enfoque de la Investigación}

\section{Método}


En la presente investigación se empleará la modalidad cuali-cuantitativa de acuerdo al paradigma critico- propositivo. Se determina cuantitativa porque confía la mediación numérica, el conteo y el empleo de la estadística descriptiva para el análisis de interpretación de resultados que permitirán verificar la hipótesis. Además, es cualitativa porque se basa en métodos de recolección de datos sin medición numérica, es decir mediante la observación para reconstruir la realidad del Coaching en el rendimiento laboral de los empleados de la Cooperativa de Ahorro y Crédito Cámara de Comercio de Ambato Ltda.

\section{Modalidad Básica de la Investigación}

Se aplicaron las siguientes modalidades de investigación:

Investigación Bibliográfica o Documental: De acuerdo con (Muñoz, 2011) se refiere a un análisis de la información escrita sobre un tema determinado tema, con el fin de entablar relaciones, diferencias, etapas, posturas o estado presente del conocimiento en relación al tema de Estudio. Las consultas documentales pueden ser de: libros, revistas, periódicos, memorias, anuarios, registros, constituciones, etc.

Investigación de Campo: Se efectúa en el lugar y tiempo en que ocurren los fenómenos objetos de estudio, es decir en la Cooperativa de Ahorro y Crédito de Comercio de Ambato Ltda. A través del contacto directo del investigador con la realidad; su finalidad es recolectar, registrar información primaria referente al problema en estudio.

\section{Tipo de investigación}

Investigación Exploratoria: Se puede determinar la aplicación de la investigación exploratoria, si se analiza el objetivo que tiene la investigación que se ha realizado, si su objetivo es examinar o explorar un tema o problema de investigación poco estudiado o que no hay sido abordado nunca se está hablando de una investigación exploratoria. (Introduccion a la investigacion de las Ciencias Sociales, 2013).

Investigación Descriptiva: Según Explorable (2013) indica que: El Diseño de investigación descriptiva es un método válido para la investigación de temas o sujetos específicos y como un antecedente a los estudios más cuantitativos. Aunque hay algunas preocupaciones razonables en relación a la validez estadística, siempre y cuando las limitaciones sean comprendidas por el investigador, este tipo de estudio representa una herramienta científica invaluable (párr. 5).

Investigación Correlacional: Para comprobar la aplicación de una investigación correlacionar, se necesita determinar que se relacionen dos o más conceptos o variables en los mismos sujetos. Es decir, correlación pudiendo determinar de qué tipo y cuál es su grado de intensidad (cuan correlacionadas están). (Introduccion a la investigacion de las Ciencias Sociales, 2013).

\section{Población y muestra}

La población de estudio está conformada de la siguiente manera entre socios y empleados tenemos una población de 10213 personas. Debido a que el segmento de la población es un 
número muy grande, es necesario calcular el tamaño de la muestra, por medio de la siguiente formula:

$$
n=\frac{N}{E^{2}(N-1)+1}
$$

\section{En donde:}

$\mathrm{n}=$ Tamaño de la muestra

$\mathrm{N}=$ Población: 10.169

$\mathrm{E}=$ error de muestreo $=5 \%=0,05$

$$
n=\frac{10.169}{0,05^{2}(10.169-1)+1}
$$

\section{Análisis de resultados}

$$
\mathrm{n}=385
$$

Análisis estadístico regresión lineal

Las preguntas que se han tomado en cuenta para aplicar la regresión lineal son:

3. ¿Le gustaría ampliar sus conocimientos dentro de la Cooperativa?

10. ¿Considera usted que desarrollar un programa de Coaching dentro de la Cooperativa ayudara a mejorar el desempeño laboral?

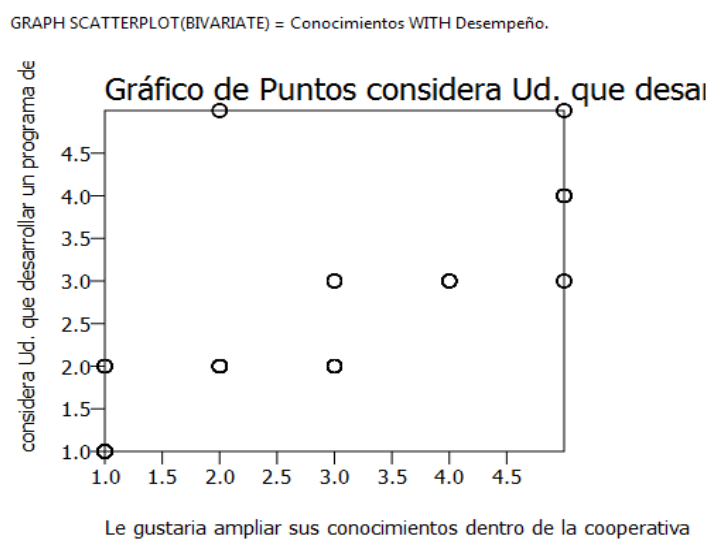

Regresion

Figura. 1. Regresion lineal

/Variables $=$ Conocimientos $\mathrm{X}$

$/$ Dependent $=$ Desempeño $\mathrm{Y}$

/Method $=$ Enter

/Stadistics $=$ Coeff R Anova

Tabla 1. Regresion Lineal

\begin{tabular}{cccc}
\hline $\boldsymbol{R}$ & $\boldsymbol{R}$. Cuadrada & R Cuadrada Ajustada & Error standard del estimador \\
\hline $\mathbf{8 8}$ & .77 & .77 & .48 \\
\hline
\end{tabular}

ANOVA del modelo (considera Ud. que desarrollar un programa de Coaching dentro de la cooperativa ayudará a mejorar el desempeño) 
Tabla 2. Anova Del Modelo

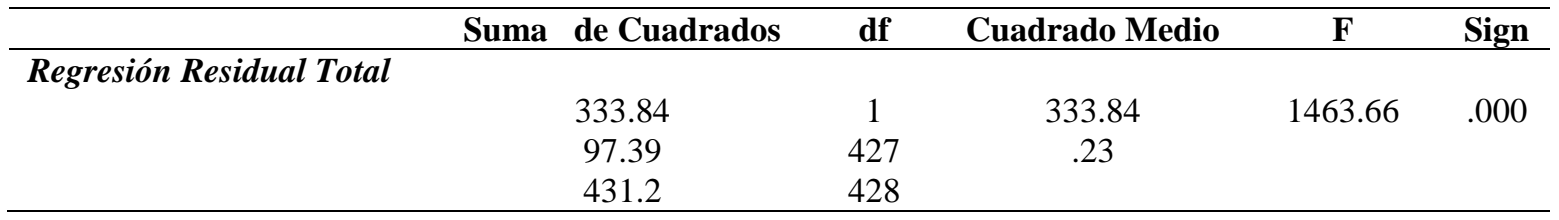

Coeficientes (considera Ud. que desarrollar un programa de Coaching dentro de la cooperativa ayudará a mejorar el desempeño)

Tabla 3. Coeficientes

\begin{tabular}{lccccc}
\hline & $\begin{array}{c}\text { Coeficientes No } \\
\text { Estandarizados }\end{array}$ & $\begin{array}{c}\text { Coeficientes } \\
\text { Estandarizados }\end{array}$ & T & Sign \\
\hline & B $\quad \begin{array}{l}\text { Error } \\
\text { Estándar }\end{array}$ & Beta & & \\
(constant)Le gustaría ampliar sus & .47 & .04 & .00 & 11.09 & .000 \\
conocimientos dentro de la Cooperativa & .69 & .02 & .88 & 38.26 & .000 \\
\hline
\end{tabular}

Análisis de los Estadísticos Encontrados

$\mathrm{R}^{2}$ Expresa la proporción de la varianza de variables de la variable dependiente que esta explicada por la variable independiente, el coeficiente de determinación toma un valor bajo (su máximo es 1 ), $\mathrm{R}^{2}$ indica que el $0.77 \%$ de la variación de desarrollar un programa de Coaching en la cooperativa (Y) esta explicada por gusto de ampliar os conocimientos dentro de la misma (X).

Coeficiente de correlación múltiple $(R)$

Interpretación:

Si $\mathrm{r}=0$ no existe correlación entre las variables

$\mathrm{Si} \pm 0,70 \leq \mathrm{r}< \pm 1,00$ existe una correlación alta

Si $\mathrm{r}=-1$ existe una correlación perfecta negativa

En nuestro caso el valor de $\mathrm{R}$ es 0.77 , lo que nos expresa que existe una correlación alta entre las variables en estudio, ampliar los conocimientos dentro de la cooperativa y desarrollar un programa de coaching que mejore el desempeño laboral.

\section{Conclusiones}

En relación al objetivo general con las encuestas realizadas a los socios y empleados de la cooperativa se puede definir que la aplicación del procedimiento del coaching contribuye al mejoramiento del desempeño laboral.

El coaching es un proceso sistemático que facilita el aprendizaje y promueve cambios cognitivos, emocionales y conductuales que expanden la capacidad de acción en función del logro de las metas propuestas (Anzonera, 2013, párr.6).

Durante nuestra investigación se ha llegado a la conclusión de que toda organización debe brindar a sus colaboradores charlas de orientación que motiven y promuevan el desarrollo de las metas colectivas tanto de los colaboradores y de la empresa fomentando un 
compromiso colectivo. Con relación a la muestra encuestada se puede analizar que la mayoría de las personas encuestadas consideran que son útiles la implementación de las charlas, capacitaciones que la empresa debe ofrecer en beneficio del talento humano. Por tanto, las charlas de orientación son útiles e importantes para los colaboradores, ya que esto les permite ampliar y desarrollar sus conocimientos y habilidades laborales, facilitándoles el cumplimiento de los objetivos y su crecimiento laboral.

Mocatero (2014) manifiesta que "Un coach es tu socio en el logro de objetivos personales. Tu entrenador en herramientas comunicacionales y de vida, que te acompaña en la generación de cambios. Sostén incondicional de éxitos y errores. Guía en desarrollo personal. Co-diseñador en la creación de nuevos proyectos. Aliento en tiempos tormentosos. Es quien te despierta cuando estás dispuesto a escuchar tu propia alarma.".

Durante nuestra investigación se ha podido observar que una parte de los colaboradores no tienen aún definidos sus objetivos personales, por lo cual el coaching será una herramienta muy importante en su desarrollo laboral que permitirá que todos los colaboradores definan bien sus objetivos personales y los trabajen en conjunto con los objetivos generales de la cooperativa, buscando siempre en desarrollo mutuo entre las partes.

Farfán (2014), sostiene que el coaching es la afinidad entre las partes (coach y coachee) "para que esta herramienta del Management (refiriéndose al coaching) sea exitosa es sumamente importante que exista química entre quien la imparte y quien la recibe".

De acuerdo a la investigación realizada, es muy importante que toda información y conocimiento que se imparte dentro de la empresa permita que tanto los colaboradores como los jefes puedan interactuar y transmitir libremente todo conocimiento que ayude en el rendimiento laboral. Toda herramienta trabajada a través del coaching permitirá a los colaboradores a mejorar laboralmente, y así mismo al mejorar laboralmente incrementaran sus conocimientos generales, permitiéndoles así una superación continua.

Hopkins (2014), sostiene que "Busca (el coaching) no sólo la transformación o la mejora continua del ejecutivo, sino un cambio trascendente o una modificación importante".

De acuerdo a la investigación realizada, el coaching no busca solamente ayudar a las personas a resolver una problemática, lo que busca es que exista un cambio importante en la vida de las personas, con lo cual poco a poco, el colaborador pueda mejorar, identificar mejor los problemas y las alternativas de solución y de esta forma poder solucionar todas las situaciones que se le presenten en beneficio colectivo tanto de la empresa como de los colaboradores. Concluye que la retención de personal no es un tema exclusivo del departamento de recursos humanos

Herrera (2014) concluye que la retención de personal no es un tema exclusivo del departamento de recursos humanos. Se trata en realidad de un problema que afecta a toda la organización y, por esta razón, es importante que sea entendido como tal, ya que todos los directivos de la organización deben estar comprometidos en la solución. 
De acuerdo a la investigación realizada opinamos que es importante que los colaboradores conozcan la trayectoria de la cooperativa para la cual laboran. Ya que eso muestra mucho interés por el lugar en donde laboran.

Las relaciones con los superiores, los compañeros y los subalternos en el trabajo pueden ser, para bien, una fuente de satisfacción y realización personal y, para mal, una rutina desgastante e incluso enfermiza. Por tal motivo es ideal que la gerencia propicie y facilite la evolución de los grupos de trabajo para que se conviertan en equipo de trabajo.(Chiavenato, 2013)

Chiavenato (2013), manifiesta que se debe elegir un estimulo adecuado para que un colaborador mejore su rendimiento. Este estímulo debe de estudiarse muy bien en base al ciclo motivacional y la pirámide de motivación.

Consideramos que un salario insuficiente desmotiva a los colaboradores de la organización. En este caso, todos los trabajadores se sienten satisfechos o muy satisfechos con su salario, pero siempre debemos recordar que el salario es una parte dentro de la motivación laboral. Es importante el reconocimiento de logro de metas porque esto influye en los compañeros de trabajo. A las personas les enorgullece darse cuenta de que su trabajo ha ayudado a alcanzar las metas. Enfatizando el logro de metas se les recuerda a los colaboradores que el éxito es posible y esto les da energía para labores futuras.

De acuerdo a la investigación que se ha realizado se puedo llegar a la conclusión de que toda cooperativa debe brindar un Programa de Coaching, debido a que es una herramienta, que permite a los colaboradores de las instituciones financieras hacer un cambio en sus perspectivas y aumentar el compromiso de responsabilidad que se traduce en mejores resultados para las mismas. Al finalizar la investigación se puede observar que los trabajadores no demuestran su compromiso e identificación con la cooperativa, debido a la demasiada rotación laboral, cuyo factor principal que motiva a renunciar a los empleados de la cooperativa es el incumplimiento del pago de horas extras, falta de bonos, incentivos, viáticos que reciben por la institución. El coaching puede dar una nueva orientación a las cooperativas, inyectándolas las energías para alcanzar las metas, incentivar el crecimiento económico y llevar al fortalecimiento de las instituciones financieras. El coaching ayuda a fortalecer el aprendizaje de los empleados de la cooperativa dándoles seguridad y confianza en lo que hacen, lo que logra que exista más productividad por parte de ellos.

\section{Acosta, \\ Bibliografía}

Acosta, Rigoberto. 2011. COACH Latinoamérica. [En línea] 2011. Http://www.coachlatinoamerica.com/blog/mindset-empresarial/conoces-las-diferencias-entrecoaching-de-negocios-y-coaching-de-vida-objetivos-personales-liderazgo-coaching-denegocios-clientes/.

Anzorena, Oscar. 2013. Coaching y aprendizaje transformacional. 2013.

Bancafacil. 2016. Bancafacil. Sitio Educativo Sbif. [En Línea] 16 Viernes De Septiembre de 2016. $\mathrm{Http} / / / \mathrm{www}$. bancafacil.cl/bancafacil/servlet/Contenido?Indice=1.5. 
Chiavenato, Idealberto. 2013. Administración de Recursos Humanos. Bogotá: Mc Graw Hill, 2013.

Entre el coaching ejecutivo y el coaching de negocios. Gil, Fernando.2015. 2015, Semana Económica, pág.párr.2.

Explorable. Explorable. [En línea] https://explorable.com/es/diseno-de-investigacion-descriptiva

Farfán. 2014. "Counching en empresas de servicios". Perú: X. $3^{\circ}$ Edición Publigrafic., 2014.

Gestionando la confianza desde el coaching organizacional como medio de prevención de conflictos. Schiavo, Valeria y López, Andrea. 2012. 2012, pág. 1.

Gonzales, Gerardo. 2014. Grandes Pymes. [En línea] 2014. Http://www.grandespymes.com.ar/2014/02/11/coaching-de-negocios-el-desarrollo-personalno-es-personal/.

Guía del Emprendor. 2016. Guia del Emprendor. [En línea] 27 de Octubre de 2016. Http://www.guiadelemprendedor.com.ar/coaching-de-negocios.html

Hall, Michael y Michael, Duval. 2010. META COACHING. MÉXICO: TRILLAS, 2010.

Herrera. 2014. Estudio exploratorio descriptivo sobre prácticas de coaching desde el punto de vista del coache. Bogotá: Universidad de Bogotá, 2014.

Hopkins. 2014. El Coaching Transformación a la mejora continua del ejecutivo. Argentina: Lantauro, 2014.

Hose, Carl. Ehow en español. [En línea] http://www.ehowenespanol.com/definicion-desempenolaboral-info_149860/.

Introducción a la investigación de las Ciencias Sociales. Cazau, Pablo. 2013. 2013, pág. 194.

La Figura del Coaching en el Ámbito Laboral. Corte, Jesús de la. 2004. 2004, pág. 5.

Mocatero. 2014. Un Coach 2014. México: Mc. Graw Hill, 2014.

Muñoz, Érica María Lara. 2011. Fundamentos de Investigación. México: Alfaomega, 2011.

Soyentrepreneur. 2013. Entrepreneur. [En línea] 15 de Mayo de 2013. Https://www.entrepreneur.com/article/265964.

Suárez, Alfonso. 2015. Qué es el Coaching. Madrid, España: s.n., 2015.

Talles, Marco. 2011. Aprende a pensar. [En línea] 28 de Junio de 2011. Http://aprendamos.aprenderapensar.net/2011/06/29/hola-mundo/.

Tovar, Manuel. 2012. El coaching en la escuela: Aplicación del coachin para los educadores. México D.F, México: Trillas, 2012. 